\title{
SMALL ISOMORPHISMS BETWEEN GROUP ALGEBRAS
}

\author{
by G. V. WOOD
}

(Received 27 June, 1989)

If $G_{1}$ and $G_{2}$ are locally compact groups and the algebras $L^{1}\left(G_{1}\right)$ and $L^{1}\left(G_{2}\right)$ are isometrically isomorphic, then $G_{1}$ and $G_{2}$ are isomorphic (Wendel, 1952, [8]). There is evidence that the following generalization of Wendel's result is true.

If $T$ is an algebra isomorphism of $L^{1}\left(G_{1}\right)$ onto $L^{1}\left(G_{2}\right)$ with $\|T\|<\sqrt{ } 2$, then $G_{1}$ and $G_{2}$ are isomorphic.

This was proved for abelian groups and for connected groups in [1], but in the general case, it is still unproved. Some partial results have been obtained. That $G_{1}$ and $G_{2}$ are isomorphic when $\|T\|<1.246$ was proved in [1]. This was improved to the condition $\|T\|<(1+\sqrt{ } 3) / 2$ in $[8]$, and the number $(1+\sqrt{ } 3) / 2$ has some special significance, as we shall see later.

In this paper, we prove the conjecture for a large class of non-abelian groups when $T$ is a $*$-isomorphism. We also show that, for groups outside this class, the existence of a *-isomorphism between their group algebras with norm $<\sqrt{2}$ means that the groups are "nearly" isomorphic. (See Propositions 14, 15, and 16). Corresponding results are also true for the algebra $M(G)$ and for $C(G)$ when $G$ is compact.

It was shown in [8] that the problem reduces to the discrete case. Let $G_{1}$ and $G_{2}$ be discrete groups and let $T$ be an algebra isomorphism of $l^{1}\left(G_{1}\right)$ and $l^{1}\left(G_{2}\right)$ with $\|T\|<\sqrt{ } 2$. Then there exists a map $t$ of $G_{1}$ into $G_{2}$ defined by the equation $T x=a t(x)+f$, where $|a|>1 / \sqrt{ } 2$. (See [1, Proposition 2.1].)

If $\|T\|<(1+\sqrt{3}) / 2$, then $t$ is a group isomorphism. (This was proved for abelian groups in [1, Theorem 2.6], and in the general case in [8, Theorem 2.2].) For $\|T\| \geqslant(1+\sqrt{ } 3) / 2$, $t$ need not be a isomorphism.

ExAmple. Let $G$ be a cyclic group of order 6 with generator $x$. Define $T x=$ $-x / 2+i \sqrt{ } 3 x^{4} / 2$, and extend to an algebra isomorphism of $\mathbb{C} G$ onto $\mathbb{C} G$. Then $\|T\|=(1+\sqrt{ } 3) / 2$, yet $t(x)=x^{4}$.

Even though $t$ need not be an isomorphism, it is always true that $t\left(x^{-1}\right)=t(x)^{-1}$. (See Lemma 2.1 in [8].)

We now assume that $T$ is a *-map. If $T x=\sum a_{i} y_{i}$, then $T x^{-1}=\sum \bar{a}_{i} y_{i}^{-1}$. It follows that $T$ is an isometry for the $l^{2}$ norm. Comparing the coefficient of the identity in $(T x)\left(T x^{-1}\right)$ gives $\sum\left|a_{i}\right|^{2}=1$. It is this property that makes the case of *-isomorphisms more tractible than the general case. This fact, together with the $\sqrt{ } 2$ bound on the norm gives inequalities for the coefficients independent of the group structure.

Lemma 1 ([6, Lemma 1]). If $\left(a_{i}\right) \in l^{1}$ with $\sum\left|a_{i}\right|=K<\sqrt{ } 2, \sum\left|a_{i}\right|^{2}=1$, and $\left|a_{1}\right| \geqslant$ $\left|a_{2}\right| \geqslant\left|a_{3}\right|, \ldots$, then

(a) $\left|a_{2}\right| \geqslant\left(1-\left|a_{1}\right|^{2}\right) /\left(K-\left|a_{1}\right|\right)$,

(b) $\left|a_{2}\right| \geqslant\left(K-\left|a_{1}\right|\right) / 2+\sqrt{ }\left(\left(1-\left|a_{1}\right|^{2}\right) / 2-\left(K-\left|a_{1}\right|\right)^{2} / 4\right)$,

whenever the expression under the square root sign is positive; i.e. when $\left|a_{1}\right| \leqslant K / 3+$ $(2 / 3) \mathrm{V}\left(\left(3-K^{2}\right) / 2\right)$,

(c) $\left|a_{3}\right| \leqslant K / 3-\sqrt{ }\left(\left(3-K^{2}\right) / 2\right) / 3$.

As in [1], we consider the two cases-whether or not $t\left(x^{2}\right)=t(x)^{2}$.

Glasgow Math. J. 33 (1991) 21-28. 
Lemma 2. If $t\left(x^{2}\right)=t(x)^{2}$ and $T x=a t(x)+f$, with $|a|>1 / \sqrt{ } 2$, then $|a|>0.907$.

Proof. Let $T x=a t(x)+b w+f$, where $|b|$ is greater than all the coefficients in $f$. We consider two cases:

(1) If $w$ commutes with $t(x)$, then the coefficient of $t(x) w$ in $T x^{2}$ has modulus $2|a b|-\|f\|_{2}^{2}=(|a|+|b|)^{2}-1$. Since this is not the largest coefficient in $T x^{2},(|a|+|b|)^{2}-$ $1<1 / \sqrt{ } 2$ i.e.

$$
|a|+|b|<\sqrt{ }(1+1 / \sqrt{ } 2)<1 \cdot 307
$$

(2) If $w$ does not commute with $t(x)$, then the coefficient of $t(x) w$ in $T x^{2}$ has modulus $>|a b|-(|a|+|b|)(\sqrt{ } 2-|a|-|b|)$. The same is true for the coefficients of $w t(x)$. Since one of these is neither the first nor second largest coefficient in $T x^{2}$, by Lemma $1(\mathrm{c})$, we have

$$
|a b|-(|a|+|b|)(\sqrt{ } 2-|a|-|b|)<1 /(3 \sqrt{ } 2) .
$$

Now $|a|^{2}+|b|^{2} \leqslant 1$ and so

$$
\left(|a|^{2}+|b|^{2}\right) / 2+|a b|-(|a|+|b|)(\sqrt{ } 2-|a|-|b|)<1 / 2+1 /(3 \sqrt{ } 2) .
$$

Putting $|a|+|b|=A$, we have

$$
A^{2} / 2-A(\sqrt{ } 2-A)<1 / 2+1 /(3 \sqrt{ } 2)
$$

i.e.

$$
3 A^{2} / 2-\sqrt{ } 2 A<1 / 2+1 /(3 \sqrt{ } 2),
$$

i.e.

or

$$
A^{2}-(2 \sqrt{ } 2) A / 3<1 / 3+\sqrt{ } 2 / 9
$$

$$
(A-\sqrt{ } 2 / 3)^{2}<(5+\sqrt{ } 2) / 9 .
$$

Hence $A<\sqrt{ } 2 / 3+\sqrt{ }(5+\sqrt{ } 2) / 3<1.316$. Thus in both cases, we have $A<1.316$, and using Lemma $1(\mathrm{~b}),|a|>0.907$, as required.

If we have this condition for all $x$ in $G_{1}$, then $t$ is a homomorphism.

THEOREM 3. If $t\left(x^{2}\right)=t(x)^{2}$, for all $x$ in $G_{1}$, then $t$ is a homomorphism.

Proof. Let $T x=a t(x)+f$ and $T y=b t(y)+g$. By Lemma 2, $|a|>0.907$ and $|b|>$ 0.907 . Hence the coefficient of $t(x) t(y)$ in $T x y$ has modulus greater than

$$
|a b|-\|f\|_{2}\|g\|_{2}=|a b|-\sqrt{ }\left(1-|a|^{2}\right)\left(1-|b|^{2}\right) .
$$

But this is greater than $(0.907)^{2}-\left(1-(0.907)^{2}\right)>0.65$. Now the largest coefficient in $T x y$ has modulus $>0.907$ by Lemma 2 . Since $(0.64)^{2}+(0.907)^{2}>1, t(x y)=t(x) t(y)$. Since this is true for all $x$ and $y, t$ is a homomorphism.

We now turn to the case when $t\left(x^{2}\right) \neq t(x)^{2}$.

THEOREM 4. If $T x=a t(x)+f$ and $t\left(x^{2}\right) \neq t(x)^{2}$, then $u=t\left(x^{2}\right) t(x)^{-2}$ has order 2, commutes with $t(x)$, and we have $T x=a t(x)+b u t(x)+g$ with $|a|+|b|>1 \cdot 29$.

Proof. Let $u=t\left(x^{2}\right) t(x)^{-2}$. Then $u t(x)^{2}$ has the largest coefficient in $T x^{2}$. Let $T x=a t(x)+b u t(x)+g$. If $u$ does not commute with $t(x)$, the coefficient of $u t(x)^{2}$ in $T x^{2}$ has modulus at most

$$
|a b|+(|a|+|b|)(\sqrt{ } 2-|a|-|b|)
$$


Since $1 / \sqrt{ } 2<|a| \leqslant 1$,

$$
\begin{aligned}
|a b|+(|a|+|b|)(\sqrt{ } 2-|a|-|b|) & <(1 / \sqrt{ } 2)|b|+(1 / \sqrt{ } 2+|b|)(1 / \sqrt{ } 2-|b|) \\
& =(1 / \sqrt{ } 2)|b|+1 / 2-|b|^{2} \\
& =5 / 8-(|b|-1 /(2 \sqrt{ } 2))^{2} \\
& <5 / 8
\end{aligned}
$$

This contradicts the fact that $u t(x)^{2}$ has the largest coefficient in $T x^{2}$. The rest of the proof now follows as in the abelian case (Lemma 2.4 of [1]). However, there is a minor error in that part of the proof that shows $u$ has order 2 . This is rectified as follows. In estimating the coefficient of $u^{-1}$, the inequality should be

$$
|a \bar{b}| \leqslant(|a|+|b|)\|f\|+\|f\|^{2},
$$

which implies that $|b|<0 \cdot 195$, but this still gives a contradiction to $|b|>0 \cdot 37$.

CoRollaRY 5. Under the hypothesis of Theorem 4, if $T x^{2}=a_{1} u t(x)^{2}+b_{1} t(x)^{2}+f_{1}$, then either $|a|+|b| \geqslant 1 \cdot 36$ or $\left|a_{1}\right|+\left|b_{1}\right| \geqslant 1 \cdot 36$.

Proof. Since $|a|+|b|>1 \cdot 29$, it is clear that $|b|$ is the second largest coefficient in $T x$. Now if $|a|+|b|<1 \cdot 36$, then, by Lemma $1(\mathrm{~b}),|a|>0.87$ and $|b|<0.49$, and so $\left|a_{1}\right| \leqslant 2|a b|+\|f\|_{2}^{2}=2|a b|+\left(1-|a|^{2}-|b|^{2}\right)=1-(|a|-|b|)^{2}<1-(0 \cdot 38)^{2}<0 \cdot 86$. Now

$$
\left|b_{1}\right| \geqslant|a|^{2}-|b|^{2}-\|f\|_{2}^{2}=2|a|^{2}-1>0 \cdot 51,
$$

so is certainly the second largest coefficient in $T x^{2}$. Thus, by Lemma 1(b) again, $\left|a_{1}\right|+\left|b_{1}\right| \geqslant 1 \cdot 36$.

We now show that only one element $u$ of order 2 can arise in this way.

Lemma 6. The set $\left[t\left(x^{2}\right) t(x)^{-2}: x\right.$ in $\left.G_{1}\right]$ contains at most one non-trivial element.

Proof. Suppose that $u=t\left(x^{2}\right) t(x)^{-2}, v=t\left(y^{2}\right) t(y)^{-2}$, where $u \neq v$, and both have order 2. Then, by Corollary 5 , we may assume that $T x=a_{1} t(x)+b_{1} t(x) u+f_{1}$, and $T y=a_{2} t(y)+b_{2} t(y) v+f_{2}$ with $\left|a_{1}\right|+\left|b_{1}\right| \geqslant 1 \cdot 36$ and $\left|a_{2}\right|+\left|b_{2}\right| \geqslant 1 \cdot 36$. Now

$$
\begin{aligned}
T x y= & a_{1} a_{2} t(x) t(y)+a_{1} b_{2} t(x) t(y) v+a_{2} b_{1} t(x) u t(y) \\
& +b_{1} b_{2} t(x) u t(y) v+\left(a_{1} t(x)+b_{1} t(x) u\right)^{*} f_{2} \\
& +f_{1}^{*}\left(a_{2} t(y)+b_{2} t(y) v\right)+f_{1}^{*} f_{2} .
\end{aligned}
$$

Since $t(x) t(y), t(x) t(y) v, t(x) u t(y)$ and $t(x) u t(y) v$ are all distinct, we have

$$
\begin{aligned}
\|T x y\| \geqslant & \left|a_{1} a_{2}\right|+\left|a_{1} b_{2}\right|+\left|a_{2} b_{2}\right|+\left|b_{1} b_{2}\right|-\left(\left|a_{1}\right|+\left|b_{1}\right|\right)\left\|f_{2}\right\| \\
& -\left(\left|a_{2}\right|+\left|b_{2}\right|\right)\left\|f_{1}\right\|-\left\|f_{1}\right\| \| f_{2}|| \\
\geqslant & (1 \cdot 36)^{2}-2(1 \cdot 36)(\sqrt{ } 2-1 \cdot 36)-(\sqrt{ } 2-1 \cdot 36)^{2} \\
= & (1 \cdot 36)^{2}-(\sqrt{ } 2-1 \cdot 36)(\sqrt{ } 2+1 \cdot 36) \\
= & 2(1.36)^{2}-2 \\
> & 1.69 .
\end{aligned}
$$

This contradicts $|T|<\sqrt{ } 2$ and so $u=v$. 
We would now like to quotient out by the subgroup $[e, u]$, but to do this we must first prove that the subgroup is normal-i.e. that $u$ commutes with all elements of $G_{2}$. We know already that $u$ commutes with $t(x)$ whenever $t\left(x^{2}\right) \neq t(x)^{2}$. We will show that $u$ commutes with $t(x)$ for all $x$, and then show that $t$ is onto. We need a refinement of Lemma 6.

Lemma 7. Let $[e, u]=\left[t\left(x^{2}\right) t(x)^{-2}: x\right.$ in $\left.G_{1}\right]$. For $y$ in $G_{1}$, let $T y=a t(y)+b t(y) v+f$, where $|a|>1 / \sqrt{ } 2$ and $b$ is the second largest coefficient. If $v \neq u$ or if $v=u$ but does not commute with $t(y)$, then $|a|+|b|<1.256$ and $|a|>0.933$.

Proof. Suppose $v \neq u$. As in Lemma 6 , choose $x$ in $G_{1}$ such that $T x=a_{1} t(x)+$ $b_{1} t(x) u+f_{1}$ with $\left|a_{1}\right|+\left|b_{1}\right|>1 \cdot 36$. If $|a|+|b|=A$, the final inequality of Lemma 6 becomes

$$
\begin{aligned}
\|T x y\| \geqslant & A(1.36)-A(\sqrt{ } 2-1 \cdot 36)-1.36(\sqrt{ } 2-A) \\
& -(\sqrt{ } 2-A)(\sqrt{ } 2-1.36) \\
= & 2 A(1.36)-2
\end{aligned}
$$

Since $\|T\|<\sqrt{ } 2$, we have $A<(2+\sqrt{ } 2) / 2 \cdot 72<1.256$. By Lemma $1(\mathrm{~b}),|a|>0.933$ as required. Now if $v=u$, but does not commute with $t(y)$, then the second largest coefficient in $T y$ cannot be both $t(y) u$ and $u t(y)$. Thus the same argument shows that in this case we also have $|a|+|b|<1.256$ and $|a|>0.933$.

THEOREM 8. $t(x) u=u t(x)$, for all $x$ in $G_{1}$.

Proof. Suppose that there exists $x$ in $G_{1}$ such that $t(x) u \neq u t(x)$ and let $T x=a t(x)+f$ with $|a|>1 / \sqrt{ } 2$. By Lemma $7,|a|>0.933$. Choose $y$ in $G_{1}$ such that $t\left(y^{2}\right) \neq t(y)^{2}$, and let $T y=a_{1} t(y)+f_{1}$ with $\left|a_{1}\right|>1 / \sqrt{ } 2$. We shall prove that $t(x y)=t(x) t(y)$. Let $T x y=$ $a_{2} t(x y)+f_{2}$ with $\left|a_{2}\right|>1 / \sqrt{ } 2$. Now the coefficient of $t(x) t(y)$ in $T x y$ has modulus greater than

$$
\left|a a_{1}\right|-\|f\|_{2}\left\|f_{1}\right\|_{2} \geqslant(1 / \sqrt{ } 2)\left(\left|a_{1}\right|-\sqrt{ }\left(1-\left|a_{1}\right|^{2}\right)\right)>0 \cdot 4 \text {. }
$$

Now if $t(x y) \neq t(x) t(y)$, then $\left|a_{2}\right| \leqslant \sqrt{ }\left(1-(0.4)^{2}\right)<0.92$. By Lemma 1 (c), the coefficient of $t(x) t(y)$ must be the second largest in Txy and so, by Lemma $7, t(x) t(y)=t(x y) u=$ $u t(x y)$. But this is a contradiction since $u$ commutes with $t(y)$, but not with $t(x)$. Hence $t(x y)=t(x) t(y)$. Applying the same argument with $x y$ in place of $x$, we obtain $t\left(x y^{2}\right)=t(x y) t(y)$, but with $y^{2}$ in place of $y$, we get $t\left(x y^{2}\right)=t(x) t\left(y^{2}\right)$. It follows that $t\left(y^{2}\right)=t(y)^{2}$, which is a contradiction. This completes the proof that $t(x) u=u t(x)$.

We now prove that $t$ is onto. We know that $t$ maps the identity $e_{1}$ of $G_{1}$ into the identity $e_{2}$ of $G_{2}$, but here is a stronger result.

LEMMA 9. If $x \neq e_{1}$ and $T x=c e_{2}+f$, then $|c|<1 /(2 \sqrt{ } 2+1)<0 \cdot 262$.

Proof. If $T x=c e_{2}+f$, then $T\left(x-c e_{1}\right)=f$ and $T\left(x-c e_{1}\right)^{n}=f^{n}$. Now $\|f\| \leqslant K-|c|$, and so $\|f\|^{n} \leqslant(K-|c|)^{n}$. On the other hand, $\left\|\left(x-c e_{1}\right)^{n}\right\| \geqslant\left(1+|c|+|c|^{2}\right)^{n / 2}$. To see this, there exists a character $\phi$ on the group generated by $x$ such that $|\phi(x)-c| \geqslant$ $\left(1+|c|+|c|^{2}\right)^{1 / 2}$. Thus

$$
\left\|T\left(x-c e_{1}\right)^{n}\right\| /\left\|\left(x-c e_{1}\right)^{n}\right\| \leqslant(K-|c|)^{n} \mid\left(1+|c|+|c|^{2}\right)^{n n}
$$


Since $T$ has a continuous inverse, this cannot tend to zero. Therefore $(K-|c|)^{2} \geqslant$ $1+|c|+|c|^{2}$ or $|c|(1+2 K) \leqslant K^{2}-1$. Hence

$$
|c| \leqslant\left(K^{2}-1\right) /(2 K+1)<1 /(2 \sqrt{ } 2+1)<0 \cdot 262 .
$$

THEOREM 10. $t$ is one-to-one and onto.

Proof. Suppose that $t$ is not one-to-one. If $x, y$ in $G_{1}$ satisfy $t(x)=t(y)=z$, then we have

$$
\begin{array}{ll}
T x=a_{1} z+f_{1} & \left(\left|a_{1}\right|>1 / \sqrt{ } 2\right), \\
T y=a_{2} z+f_{2} & \left(\left|a_{2}\right|>1 / \sqrt{ } 2\right) .
\end{array}
$$

We consider first the case when $x$ and $y$ commute. Then

Now

$$
\begin{aligned}
T\left(a_{2} x-a_{1} y\right) & =a_{2} f_{1}-a_{1} f_{2}, \\
T\left(a_{2} x-a_{1} y\right)^{n} & =\left(a_{2} f_{1}-a_{1} f_{2}\right)^{n} .
\end{aligned}
$$

$$
\begin{aligned}
\| a_{2} f_{1}-a_{1} f_{2}|| & \leqslant\left|a_{2}\right|\left(K-\left|a_{1}\right|\right)+\left|a_{1}\right|\left(K-\left|a_{2}\right|\right) \\
& \leqslant K\left(\left|a_{1}\right|+\left|a_{2}\right|\right)-2\left|a_{1}\right|\left|a_{2}\right|<1
\end{aligned}
$$

since $\left|a_{1}\right|>1 / \sqrt{ } 2,\left|a_{2}\right|>1 / \sqrt{ } 2$ and $K<\sqrt{ } 2$. Also

as in Lemma 9. Thus

$$
\begin{aligned}
\left\|\left(a_{2} x-a_{1} y\right)^{n}\right\| & =\left\|\left(a_{2} e_{1}-a_{1} x^{-1} y\right)^{n}\right\| \\
& \geqslant\left|a_{2}\right|^{n}\left(1+\left|a_{1} / a_{2}\right|+\left|a_{1} / a_{2}\right|^{2}\right)^{n / 2},
\end{aligned}
$$

$$
\left|\left(a_{2} x-a_{1} y\right)^{n}\right| \geqslant\left(\left|a_{1}\right|^{2}+\left|a_{1} a_{2}\right|+\left|a_{2}\right|^{2}\right)^{n / 2} \geqslant 1 .
$$

This again contradicts the boundedness of $T^{-1}$.

If $x$ and $y$ do not commute, we have that $T y^{-1}=\bar{a}_{2} z^{-1}+f_{2}^{*}$. Thus $T x y^{-1}=$ $\left(a_{1} z+f_{1}\right)^{*}\left(\bar{a}_{2} z^{-1}+f_{2}^{*}\right)$.

By Lemma 9 , the coefficient of $e_{2}$ in $T x y^{-1}$ has modulus less than $1 /(2 \sqrt{ } 2+1)$. We have $\left|a_{1} a_{2}\right|-\left\|f_{1}\right\|\left\|f_{2}\right\| \leqslant 1 /(2 \sqrt{ } 2+1)$. Therefore

$$
\begin{gathered}
\left|a_{1} a_{2}\right|-\left(\sqrt{ } 2-\left|a_{1}\right|\right)\left(\sqrt{ } 2-\left|a_{2}\right|\right) \leqslant 1 /(2 \sqrt{ } 2+1), \\
\left|a_{1}\right|+\left|a_{2}\right| \leqslant(4 \sqrt{ } 2+3) /(4+\sqrt{ } 2)<1 \cdot 6
\end{gathered}
$$

It follows from Lemma 1(b), that $\left|a_{1}\right|<0.9$ and $\left|a_{2}\right|<0.9$, and so, by Lemma 2 , $t\left(x^{2}\right) \neq t(x)^{2}$ and $t\left(y^{2}\right) \neq t(y)^{2}$. By Theorem 4, we have $T x=a_{1} z+b_{1} z u+g_{1}$, with $\left|a_{1}\right|+\left|b_{1}\right|>1 \cdot 29$, and $T y=a_{2} z+b_{2} z u+g_{2}$, with $\left|a_{2}\right|+\left|b_{2}\right|>1 \cdot 29$. Now

$$
T x y^{-1}=\left(a_{1} \bar{a}_{2}+b_{1} \bar{b}_{2}\right) e+\left(a_{1} \bar{b}_{2}+\bar{a}_{2} b_{1}\right) u+h,
$$

where

$$
\begin{aligned}
\|h\| & \leqslant\left|g_{1}\right|\left(\left|a_{1}\right|+\left|b_{2}\right|\right)+\left|g_{2}\right|\left(\left|a_{1}\right|+\left|b_{2}\right|\right)+\left\|g_{1}\right\|\left\|g_{2}\right\| \\
& \leqslant K\left(\left|g_{1}\right|+\left|g_{2}\right|\right)-\left\|g_{1}\right\| \| g_{2}|| \\
& <\sqrt{ } 2(\sqrt{ } 2-1 \cdot 29)-(\sqrt{ } 2-1 \cdot 29)(\sqrt{ } 2-1 \cdot 29) \\
& =1.29(\sqrt{ } 2-1.29)<0.17 .
\end{aligned}
$$


Thus $t\left(x y^{-1}\right)=u$ and has coefficient with modulus greater than $0 \cdot 933$. This follows from Lemma 7 if the second coefficient is not that of $e_{2}$, and if it is, it is necessarily less than 0.262 . Now, by Lemma 1 (b), the biggest coefficient has modulus greater than 0.94 . Similarly $t\left(y^{-1} x\right)=u$ and has coefficient with modulus greater than 0.933 . Now $x y^{-1} \neq y^{-1} x$, since $x$ and $y$ do not commute. Repeating the argument with $x y^{-1}$ and $y^{-1} x$ in place of $x$ and $y$, we obtain a contradiction, since the sum of coefficients is less than 1.6. This completes the proof that $t$ is one-to-one.

To show that $t$ is onto, let $K=t\left(G_{1}\right)$ and $P$ the linear projection of $l_{1}\left(G_{1}\right)$ onto $l_{1}(K)$. Define $S x=a(x) t(x)$ where $T x=a(x) t(x)+f$ and $|a(x)|>1 / \sqrt{ } 2$. Extend $S$ linearly to a map from $l_{1}\left(G_{1}\right)$ to $l_{1}(K)$. Then $\|T-S\| / 1 / \sqrt{ } 2$, so that $S$ is invertible with $\left\|S^{-1}\right\| \leqslant \sqrt{ } 2$. $P S=S$ and so

$$
\left\|P T S^{-1}-I\right\|=\left\|P(T-S) S^{-1}\right\|<(1 / \sqrt{ } 2) \sqrt{ } 2=1 .
$$

Thus $P T S^{-1}$ is invertible. In particular $P$ is invertible and $K=G_{2}$.

Remark. It seems likely that $\left|(x-c y)^{n}\right| \geqslant 1$ for all $n$, even when $x$ and $y$ do not commute. If this were true, the above proof would be considerably shortened.

We have proved the main theorem.

THEOREM 11. Let $G_{1}$ and $G_{2}$ be groups and $T$ a *-isomorphism of $l_{1}\left(G_{1}\right)$ onto $l_{1}\left(G_{2}\right)$ with $|T|<\sqrt{ } 2$. Then either $G_{1}$ and $G_{2}$ are isomorphic, or there exist elements $v$ in $G_{1}$ and $u$ in $G_{2}$ both of order 2 and a map $t: G_{1}$ to $G_{2}$, such that

(i) $t$ is a bijection preserving inverses,

(ii) $t(v)=u$, and $t: G_{1}$ onto $G_{2} /[e, u]$ is a homomorphism.

Using the techniques for abelian groups contained in [1], we can obtain the following result.

THEOREM 12. Under the hypothesis of Theorem 11, if $u$ does not belong to the commutator subgroup of $G_{2}$, then $G_{1}$ and $G_{2}$ are isomorphic.

Proof. If $I_{2}$ is the identity character on $G_{2}$, then $I_{2} \circ T$ is a character on $G_{1}$. By multiplying $T$ by the inverse of this character, we may assume that $I_{2} \circ T=I_{1}$.

If $u$ does not belong to the commutator subgroup of $G_{2}$, there exists a character $\psi$ with $\psi(u)=-1$. Then the composition $\psi \circ T$ is a character on $G_{1}$, and since $t(x y)=$ $t(x) t(y)$ or $t(x) t(y) u$, we have $\psi(t(x y))= \pm \psi(t(x)) \psi(t(y))$. Thus $(\psi \circ t)^{2}$ is also a character on $G_{1}$. Define $\varphi=(\psi \circ t)^{-1}(\psi \circ T)$. Then $\varphi^{2}$ is a character on $G_{1}$. We show that $\varphi^{2}$ has odd order.

If $\varphi^{2}$ does not have odd order, there exists $x$ in $G_{1}$ such that $\varphi^{2}(x)$ is arbitrarily close to -1 . Thus, given $\varepsilon>0$, there exists $x$ in $G_{1}$ such that $|\varphi(x)+i|<\varepsilon$. If

$$
T x=a t(x)+b t(x) u+\sum c_{i} y_{i}
$$

then

$$
\varphi(x)=a-b+\sum c_{i} \varphi(t(x))^{-1} \varphi\left(y_{i}\right)
$$

Thus we have $a+b+\sum c_{i}=1$ (since $I_{2} \circ T=I_{1}$ ), and

$$
\left|a-b+\sum c_{i} \varphi\left(t(x)^{-1} y_{i}\right)+i\right|<\varepsilon .
$$


Substituting for $a$, we obtain

In particular,

$$
\left|1+i-2 b+\sum c_{i}\left(\varphi\left(t(x)^{-1} y_{i}\right)-1\right)\right|<\varepsilon .
$$

$$
|b|>|1+i| / 2-\sum\left|c_{i}\right|-\varepsilon / 2
$$

Since $a>1 / \sqrt{ } 2,|a|+|b|+\sum\left|c_{i}\right|>\sqrt{ } 2-\varepsilon / 2$, which is a contradiction.

Thus $\varphi^{2}$ has odd order, $n$ say. Let $\theta=\varphi^{n+1}$, another character on $G_{1}$, with $\theta(x)= \pm \varphi(x)$. Define $s: G_{1}$ to $G_{2}$ by

$$
\begin{array}{ccc}
s(x)=t(x) & \text { if } \quad \theta(x)=\varphi(x), \\
s(x)=t(x) u & \text { if } \quad \theta(x)=-\varphi(x) .
\end{array}
$$

Then $s$ is a homomorphism since $\varphi$ is, and since $t$ is injective and onto, $s$ is also.

This gives us the main theorem.

THEOREM 13. If $T$ is a *-isomorphism of $l_{1}\left(G_{1}\right)$ onto $l_{1}\left(G_{2}\right)$ with $\|T\|<\sqrt{2}$, and if $G_{1}$ (or $G_{2}$ ) does not contain a central element of order 2 in the commutator subgroup, then $G_{1}$ and $G_{2}$ are isomorphic.

If $G_{1}$ has a central element of order 2 in the commutator subgroup, then the map $t$ in Theorem 11 has the following two additional properties.

Proposition 14. $t$ maps the centre of $G_{1}$ into the centre of $G_{2}$.

Proof. If $x$ is in the centre of $G_{1}$, it is in the centre of $l_{1}\left(G_{1}\right)$, and hence $T x$ is in the centre of $l_{1}\left(G_{2}\right)$. Therefore if $T x=a t(x)+f$, with $|a|>1 / \sqrt{ } 2$, then for each $y$ in $G_{2}$,

$$
T x=y^{-1}(T x) y=a y^{-1} t(x) y+y^{-1} f y .
$$

No coefficient in $y^{-1} f y$ can have modulus greater than $1 / \sqrt{ } 2$, and so $y^{-1} t(x) y=t(x)$ and $t(x)$ belongs to the centre of $G_{2}$.

In fact, it can be shown, using similar techniques to those in [1, Theorem 3.4], that on the centre $Z_{1}$ of $G_{1}$ either $T$ has the form $T x=\psi(x) t(x)$, with $t$ an isomorphism and $\psi$ a character on $Z_{1}$, or the form

$$
T x=\psi(x)[((1+\theta(x)) / 2) s(x)+((1-\theta(x)) / 2) s(x) u],
$$

where $\psi, \theta$ are characters on $Z_{1}$ with $\theta$ of odd order, and $s$ is an isomorphism.

PROPOSITION 15. $t$ maps the commutator subgroup of $G_{1}$ into the commutator subgroup of $G_{2}$.

Proof. $t\left(x y x^{-1} y^{-1}\right)$ is either $t(x) t(y) t(x)^{-1} t(y)^{-1}$ or $t(x) t(y) t(x)^{-1} t(y)^{-1} u$, both of which are in the commutator subgroup of $G_{2}$.

Under these circumstances, we also have the following result.

Proposition 16. $G_{1}$ and $G_{2}$ have the same number of elements of each order.

Proof. Let $\bar{x}$ be the image of $x$ under the quotient map $G_{1} \rightarrow G_{1} /[e, v]$. If $\bar{x}$ has odd order $n$, then one of $x$ and $x v$ has order $n$, the other $2 n$. $\overline{t(x)}$ also has order $n$, and so, of the elements $t(x)$ and $t(x) u$, one will have order $n$, the other $2 n$. 
We next consider elements of order 2 . We need to show that

$$
x^{2}=e_{1} \Leftrightarrow t(x)^{2}=e_{2}
$$

If the implication in either direction is false, then $t\left(x^{2}\right) \neq t(x)^{2}$. But, if $T x=a t(x)+f$, the coefficient of $t(x)^{2}$ in $T x^{2}$ has modulus $>|a|-\|f\|^{2}>0$, which gives a contradiction.

Now suppose that $x$ and $t(x)$ have order $2 n$. For the result to be false, one of two things must happen.

(a) $x$ and $x v$ have order $2 n$ (i.e. $x^{2 n} \neq v$ ) and $t(x)$ and $t(x) u$ have order $4 n$ (i.e. $\left.t(x)^{2 n}=u\right)$. But then $\left(x^{n}\right)^{2}=e_{1}$, yet $\left(t\left(x^{n}\right)\right)^{2}=u$, which contradicts $(*)$,

(b) $x$ and $x v$ have order $4 n\left(x^{2 n}=v\right)$ and $t(x)$ and $t(x) u$ have order $2^{n}\left(t(x)^{2 n} \neq u\right)$. Then $\left(x^{n}\right)^{2}=v$, but $\left(t(x)^{n}\right)^{2}=e_{2}$. This also contradicts $(*)$.

Whether these conditions in themselves mean that the groups are isomorphic is not clear. Using the book [4] it is possible, though very tedious, to confirm that no counterexample exists with groups of order up to 32 .

The corresponding results for locally compact groups follows easily from the discrete case. (See [1], [3], and [8] for the details.)

THEOREM 17. Let $T$ be a *-isomorphism of $L^{1}\left(G_{1}\right)$ onto $L^{1}\left(G_{2}\right),\left[M\left(G_{1}\right)\right.$ onto $\left.M\left(G_{2}\right)\right]$ satisfying $\|T\|<\sqrt{ } 2$. If $G_{1}$ (or $G_{2}$ ) does not contain a central element of order 2 in the commutator subgroup, then $G_{1}$ and $G_{2}$ are isomorphic.

THEOREM 18. Let $G_{1}$ and $G_{2}$ be compact groups without central elements of order 2 in the commutator subgroup. If $T$ is a *-isomorphism of $C\left(G_{1}\right)$ onto $C\left(G_{2}\right),\left[L^{\infty}\left(G_{1}\right)\right.$ onto $\left.L^{\infty}\left(G_{2}\right)\right]$, satisfying $\|T\|<\sqrt{ } 2$, then $G_{1}$ and $G_{2}$ are isomorphic.

\section{REFERENCES}

1. N. J. Kalton and G. V. Wood, Homomorphisms of group algebras with norm less than $\sqrt{2}$, Pacific J. Math. 62 (1976), 439-460.

2. W. Rudin, Fourier analysis on groups (Interscience, 1960).

3. R. S. Strichartz, Isomorphism of group algebras, Proc. Amer. Math. Soc. 17 (1966), $858-862$.

4. A. D. Thomas and G. V. Wood, Group Tables (Shiva, 1981).

5. J. G. Wendel, Left centralizers and isomorphisms of group algebras, Pacific J. Math. 2 (1952), 251-261.

6. G. V. Wood, Distance between group algebras, Proc. Roy. Irish Acad. Sect. A 76 (1976), $339-342$.

7. G. V. Wood, Measures with bounded powers on locally compact groups, Trans. Amer. Math. Soc. 268 (1981), 187-209.

8. G. V. Wood, Isomorphisms of group algebras, Bull. London Math. Soc. 15 (1983), $247-252$.

Department of Mathematics and Computer Science

University College of Swansea

SWANSEA SA2 8PP

WALES 